\title{
Post-discharge Telemonitoring of Physical Activity, Vital Signs, and Patient-Reported Symptoms in Older Patients Undergoing Cancer Surgery
}

\author{
Leonie T. Jonker, $\mathrm{MD}^{1,2}$, Maarten M. H. Lahr, $\mathrm{PhD}^{2}$, Maaike H. M. Oonk, MD, $\mathrm{PhD}^{3}$, Geertruida H. de Bock, \\ $\mathrm{PhD}^{2}$, and Barbara L. van Leeuwen, MD, $\mathrm{PhD}^{1}$ \\ ${ }^{1}$ Department of Surgical Oncology, University of Groningen, University Medical Centre Groningen, Groningen, The \\ Netherlands; ${ }^{2}$ Department of Epidemiology, University of Groningen, University Medical Centre Groningen, Groningen, \\ The Netherlands; ${ }^{3}$ Department of Obstetrics and Gynecology, University of Groningen, University Medical Centre \\ Groningen, Groningen, The Netherlands
}

\begin{abstract}
Background. Postoperative home monitoring could potentially detect complications early, but evidence in oncogeriatric surgery is scarce. Therefore, we evaluated whether post-discharge physical activity, vital signs, and patient-reported symptoms are related to post-discharge complications and hospital readmissions in older patients undergoing cancer surgery.

Methods. In this observational cohort study, we monitored older patients ( $\geq 65$ years of age) undergoing cancer surgery, for 2 weeks post-discharge using tablet-based applications and connected devices. Outcome measures were post-discharge complications and readmissions; physical activity and patient-reported symptoms over time; and threshold violations for physical activity (step count $<1000$ steps/day), vital signs (temperature $<36^{\circ} \mathrm{C}$ or $>38^{\circ} \mathrm{C}$; blood pressure $<100 / 60 \mathrm{mmHg}$ or $>150 /$ $100 \mathrm{mmHg}$; heart rate $<50 \mathrm{bpm}$ or $>100 \mathrm{bpm}$; weight $-5 \%$ or $+5 \%$ of weight at discharge); and patient-reported
\end{abstract}

Supplementary Information The online version contains supplementary material available at https://doi.org/10.1245/s10434021-09707-3.

(C) The Author(s) 2021

First Received: 12 October 2020

Accepted: 22 January 2021;

Published Online: 27 February 2021

L. T. Jonker, MD

e-mail: 1.t.jonker@umcg.nl symptoms (pain score greater than the previous day; presence of dyspnea, vomiting, dizziness, fever).

Results. Of 58 patients (mean age 72 years), 24 developed a post-discharge complication and 13 were readmitted. Measured parameters indicated 392 threshold violations out of 5379 measurements (7.3\%) in 40 patients, mostly because of physical inactivity. Patients with readmissions had lower physical activity at discharge and at day 9 after discharge and violated a physical activity threshold more often. Patients with post-discharge complications had a higher median pain score compared with patients without these adverse events. No differences in threshold violations of other parameters were observed between patients with and without post-discharge complications and readmissions.

Conclusion. Our results show the potential of telemonitoring older patients after cancer surgery but confirm that detecting post-discharge complications is complex and multifactorial.

\section{INTRODUCTION}

Cancer imposes a large burden on global health, predominantly because of the aging population. ${ }^{1}$ In 2018 , more than half of new cancer cases and almost two-thirds of cancer deaths occurred in adults aged 65 years and older. ${ }^{2}$ Surgery is often required as a part of the curative treatment of patients with a solid tumor. ${ }^{3}$ Comorbidity and frailty (age-related physiological decline of multiple functions) are common in older patients and increase the risk of developing postoperative complications and being 
readmitted. ${ }^{4}$ Especially for older patients, postoperative complications and unplanned hospital readmissions have a large impact on their functional recovery, quality of life, and mortality. ${ }^{5}$

Several interventions aimed at decreasing postoperative adverse events have been implemented in oncogeriatric surgery, such as geriatric assessments, preoperative optimization of modifiable risk factors, minimally invasive surgical techniques, and enhanced recovery after surgery programs. $^{3}$ As a result of these interventions, as well as requirements to decrease health care costs and increase capacity, the length of hospital stay (LOS) has been significantly reduced. ${ }^{6,7}$ With the shortening of LOS, late complications such as surgical site, urinary tract, and respiratory infections and venous thromboembolic complications can occur in the period after hospital discharge. ${ }^{8,9}$ Data on post-discharge complications following oncogeriatric surgery and the circumstances at the time of their occurrence are limited. ${ }^{8}$ Identifying deviations in postoperative recovery at home could possibly support early detection of post-discharge complications, reduce their impact, or even prevent unplanned hospital readmissions. ${ }^{9,10}$

Home remote monitoring, or at-home telemonitoring, has been used in a few studies following oncological surgery, to monitor patients' physical activity, vital signs, and well-being using various types of electronic wearables, activity trackers, mobile applications, symptom surveys, and systems supporting video consultation. ${ }^{11-16}$ Although these studies demonstrate that the use of a home monitoring system after oncological surgery is feasible, its effect on clinical outcomes has not yet been demonstrated. In addition, telemonitoring studies focusing on older surgical patients are limited. ${ }^{17,18}$ To assess the effectiveness of remote home monitoring in the detection of deviations in postoperative recovery after oncogeriatric surgery, we first need to collect telemonitoring data of oncogeriatric patients with a high risk of postoperative adverse events.

Therefore, we conducted an observational cohort study with the aim of monitoring physical activity, vital signs, and patient-reported symptoms of older patients after hospital discharge following oncological surgery. To do so, we compared characteristics and home monitoring data between groups of patients with and without post-discharge complications and with and without hospital readmissions.

\section{METHODS}

\section{Study Design and Participants}

This was a prospective analysis from a single-center observational study with perioperative remote home monitoring of older patients after hospital discharge following oncological surgery in an academic teaching hospital in the north of The Netherlands. Results regarding acceptability and usability of remote home monitoring ${ }^{19}$ and postoperative recovery of physical activity ${ }^{20}$ of the first 50 patients of this cohort have previously been published, as well as the results of the recruitment process of 151 patients of the current cohort. ${ }^{21}$ Patients were eligible for inclusion if they were aged 65 years and older, were scheduled for surgical resection of a solid malignant tumor in the Department of Surgical Oncology or Department of Gynecological Oncology, and had internet access at home. Exclusion criteria were cancellation of surgery, emergency surgery, or perceived incapability to use components of the remote home monitoring system due to contact dermatitis, insufficient understanding of the Dutch language, or severe auditory, visual, cognitive, or ambulatory impairment. The local Medical Ethics Committee approved the study (local registration: 2017/286; Netherlands trial registration: NL8253).

\section{Remote Home Monitoring}

Participants' physical activity, vital signs, and patientreported symptoms were measured using commercially available monitoring devices and electronic questionnaires connected to a remote home monitoring system developed within the European Union-funded Connecare consortium (Project Grant Number: 689802). The Connecare system consists of a tablet-based health application for patients, called the self-management system (SMS) and a web-based self-adaptive case management system (SACM) for the care professional. Monitoring data were visible to patients on the SMS and regularly checked by the case manager (research physician) on the SACM. Data were not monitored in real time. Patients were contacted by telephone if data were missing or measurements were outside set values (threshold violations), to provide technical assistance or to obtain additional information regarding parameter deviations. If deemed necessary by the case manager, the treating physician could be contacted. Physical activity was monitored in every patient from the start of the study, and vital signs and patient-reported symptoms were monitored in a subset of patients as the IT system was tested and further developed during study implementation. ${ }^{19}$

\section{Physical Activity}

At preoperative baseline assessment, participants were instructed to wear a commercially available accelerometerbased wearable activity monitor (Fitbit Charge 2, Fitbit Inc., San Francisco, CA, USA). Daily step count was measured preoperatively, in the waiting time between 
baseline assessment until surgery, and postoperatively during hospital admission on the surgical ward and at home up until 3 months after surgery. Data were transferred via Bluetooth from the activity monitor to the tablet-based Fitbit application and Connecare application. A step count below 1000 was considered a threshold violation, but no step goal was provided to the patient. ${ }^{22}$

\section{Vital Signs}

A subset of the participants was discharged with additional commercially available monitoring devices (Nokia Withings, Issy-les-Moulineaux, France; Connecare SMS) to measure their vital signs every morning for 14 days postdischarge, i.e. temperature, blood pressure, heart rate, and weight. Vital signs were considered abnormal if the temperature was $<36 \circ \mathrm{C}$ or $>38^{\circ} \mathrm{C}$, blood pressure was $<100$ / $60 \mathrm{mmHg}$ or $>150 / 100 \mathrm{mmHg}$, heart rate was $<50$ or $>100$, or weight was $-5 \%$ or $+5 \%$ of weight at hospital discharge.

\section{Patient-Reported Symptoms}

A subset of the participants was asked to complete two electronic health questionnaires in the Connecare application once daily for 14 days post-discharge. The first questionnaire measured pain perception using a horizontal visual analog scale linked to a numerical rating scale, with 0 being 'no pain' and 10 being 'the worst pain imaginable'. ${ }^{23}$ We considered a pain score higher than that of the previous day to be a threshold violation. The second questionnaire was a post-surgical health questionnaire to assess patient-reported symptoms. This consisted of 12 yes/ no questions about the presence of problems that might indicate potential complications, regarding (1) breathing, (2) vomiting, (3) dizziness, (4) eating, (5) drinking, (6) urinating, (7) defecating, (8) mobility, (9) fever, (10) resting and sleeping, (11) bathing and washing, and (12) getting (un)dressed. Problems with breathing, vomiting, dizziness, or fever were considered to be alarming symptoms and were counted as threshold violations.

\section{Data Collection}

Patient characteristics on comorbidity, frailty (Groningen Frailty Indicator ${ }^{24}$ ), (instrumental) activities of daily living, ${ }^{25,26}$ nutritional status (Short-Form Mini-Nutritional Assessment ${ }^{27}$ ), and mental status (Hospital Anxiety and Depression Scale ${ }^{28}$ ) were collected at the face-to-face baseline assessments. Clinical and surgical data were collected from medical records, including in-hospital and post-discharge complications within 90 days after surgery (as classified by the Clavien-Dindo classification ${ }^{29}$ and the
Comprehensive Complication Index ${ }^{30}$ ), hospital readmission within 90 days after surgery, and timing of postdischarge complications and hospital readmissions. Data that deviated from the post-discharge course were complemented with information gathered by telephone during monitoring and at the 3-month follow-up assessment. Deviations from a normal postoperative course that resulted in consultation with a health care professional but did not require treatment were classified as Clavien-Dindo grade 0 .

\section{Outcome Measures}

Outcome measures were post-discharge complications and hospital readmissions, physical activity and symptoms over 14 post-discharge days, and threshold violations of physical activity, vital signs (temperature, blood pressure, heart rate, weight), and patient-reported symptoms.

\section{Statistical Analysis}

Descriptive statistics were used to present baseline and surgery characteristics for patients with and without postdischarge complications and readmissions. Comparison between groups were performed using the independent Student's $t$-test for continuous parametric data, the MannWhitney $U$ test for non-parametric data, and the Fisher's exact test for categorical data. We presented physical activity and patient-reported symptoms over 14 days. The total of performed measurements and threshold violations per parameter (physical activity, vital signs, and patientreported symptoms) were presented from the first 14 days after discharge, until hospital readmission, or until study dropout. For physical activity, we also analyzed data from the day of hospital discharge (day 0) and the day before hospital discharge (day -1 ). The total number of threshold violations per measured parameter are presented, as well as the percentage of patients who experienced one or more threshold violations. We compared physical activity, patient-reported symptoms, and the percentage of patients who experienced one or more threshold violations between the subgroups with/without post-discharge complications and with/without hospital readmissions. A $p$-value lower than 0.05 was considered statistically significant. Data were analyzed using SPSS statistics version 23 (IBM Corporation, Armonk, NY, USA). 


\section{RESULTS}

\section{Enrollment and Dropout}

In the period from May 2018 to March 2020, 65 of 130 eligible patients consented to participate in our study. The main reasons for refusal and ineligibility have been extensively described previously. ${ }^{21}$ After informed consent was obtained, seven patients were excluded from the study because of cancellation of surgery $(n=4)$, missing baseline assessment after rescheduling of surgery $(n=2)$, or regulations due to the coronavirus disease 2019 (COVID19) outbreak $(n=1)$ (Fig. 1). Thus, a total of 58 patients were included in this analysis. After inclusion, 2 patients died and 13 patients withdrew from the study because of the high burden of disease, surgery, or complications in combination with study participation.

\section{Patient Characteristics}

The 58 included patients had a mean age of $72 \pm 5$ years, and $38(66 \%)$ were male. The majority of patients underwent surgery because of a gastrointestinal malignancy $(n=43,74 \%)$, gynecological malignancy $(n=5$, $9 \%)$, or sarcoma $(n=4,7 \%)$, with the tumor being intracavitary in $49(85 \%)$ patients. A detailed list of operations performed is presented in electronic supplementary Table A. The median LOS was 8.5 days (interquartile range [IQR] 4.3-19.8). The characteristics of patients with and without post-discharge complications are presented in Table 1.

\section{Postoperative Adverse Events}

A total of 40/58 (69\%) patients developed a complication within 90 days after surgery: 16 only in-hospital, 11 both in-hospital and post-discharge, and 13 only post-discharge (Fig. 1). Two patients died during hospital admission. Compared with patients without post-discharge complications $(n=32)$, patients with post-discharge complications $(n=24)$ had undergone open surgery more often than laparoscopic or robotic surgery $(91.7 \%$ vs. $68.8 \% ; p=0.04$ ) (Table 1 ). The 13 patients who were readmitted had similar patient and surgery characteristics compared with patients who were not readmitted, and they also experienced in-hospital complications more often (9 [69\%] vs. 16 [37\%]; $p=0.04)$. Table 2 demonstrates that the majority of complications were infectious $(n=13$, $54 \%)$. Most first complications $(n=17,71 \%)$ and hospital readmissions $(n=8,62 \%)$ occurred within 2 weeks after discharge.

\section{Remote Home-Monitoring Results}

Of a total of 5379 measurements that were performed 2 weeks post-discharge, 392 measurements in 40/49 (82\%) patients violated the threshold. Most threshold violations

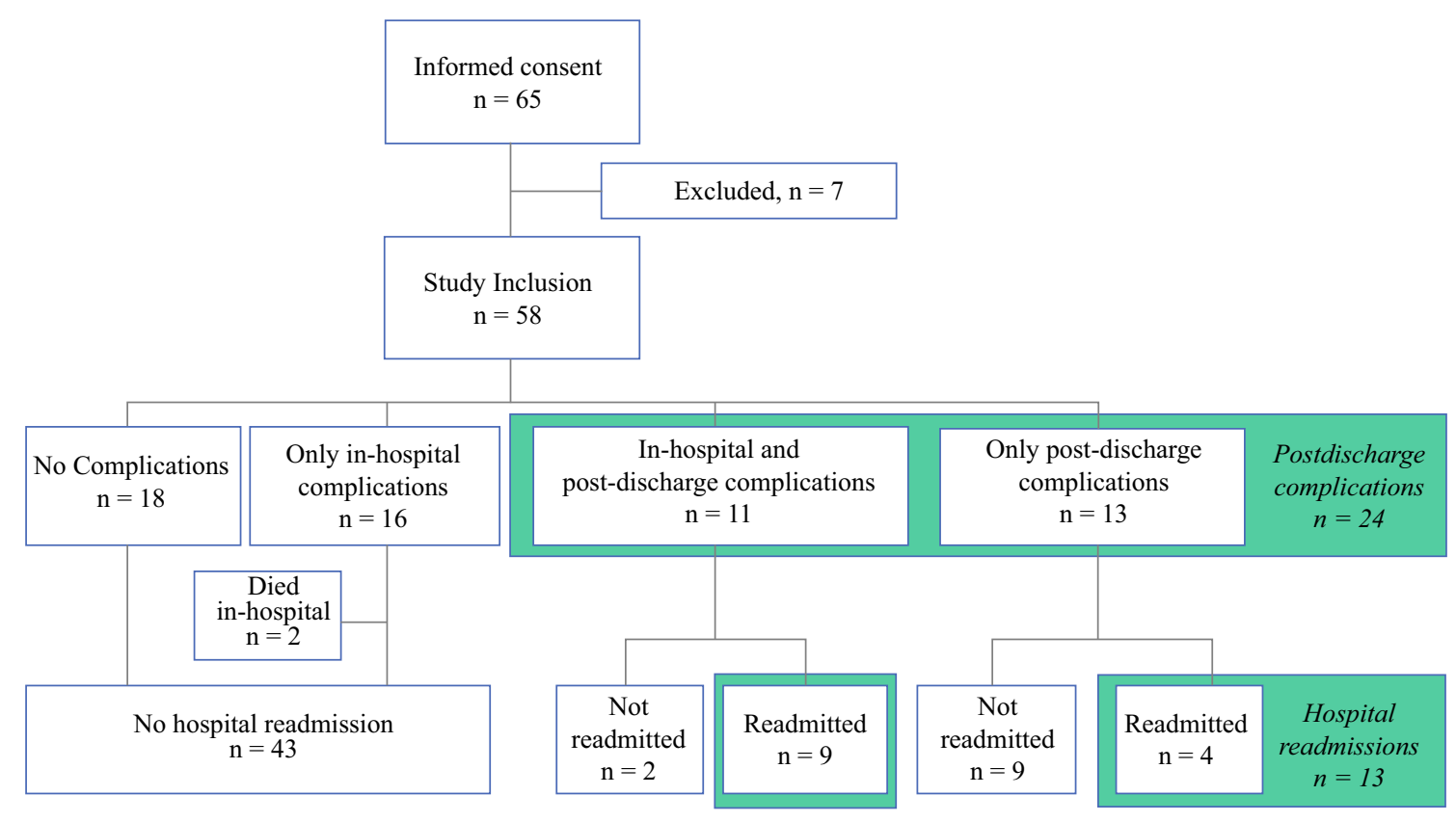

FIG. 1. Patients with in-hospital complications, post-discharge complications, and hospital readmissions. After informed consent was obtained, seven patients were excluded from the study because of cancellation of surgery $(n=4)$, missing baseline assessment after rescheduling of surgery $(n=2)$, or regulations regarding the COVID19 outbreak $(n=1)$. COVID-19 coronavirus disease 2019 
TABLE 1. Characteristics of patients with and without post-discharge complications

\begin{tabular}{|c|c|c|c|}
\hline & $\begin{array}{l}\text { Patients with post-discharge } \\
\text { complications }[n=24]\end{array}$ & $\begin{array}{l}\text { Patients without post-discharge } \\
\text { complications }[n=32]\end{array}$ & $p$-Value \\
\hline Mean age, years (SD) & $72.9(4.4)$ & $72.1(5.3)$ & 0.45 \\
\hline \multicolumn{4}{|l|}{ Gender } \\
\hline Male & $15(62.5)$ & $21(65.6)$ & \\
\hline Female & $9(37.5)$ & $11(34.4)$ & 0.75 \\
\hline \multicolumn{4}{|l|}{ ASA classification } \\
\hline $1-2$ & $2(87.5)$ & $25(78.1)$ & \\
\hline $3-4$ & $3(12.5)$ & $7(21.9)$ & 0.37 \\
\hline Median Charlson Comorbidity Index (IQR) & $3.0(2.0-6.8)$ & $5.0(2.0-6.0)$ & 0.37 \\
\hline \multicolumn{4}{|l|}{ Location of surgery } \\
\hline Intracavitary & $20(83.3)$ & $27(84.8)$ & \\
\hline Superficial & $4(16.7)$ & $5(15.6)$ & 1.00 \\
\hline \multicolumn{4}{|l|}{ Surgical technique } \\
\hline Open & $22(91.7)$ & $22(68.8)$ & \\
\hline Scopic & $2(8.3)$ & $10(31.3)$ & $0.04 *$ \\
\hline Median anesthesia time, $\min (\mathrm{IQR})$ & $378(187-475)$ & $299(180-476)$ & 0.56 \\
\hline Median surgical blood loss, mL (IQR) & $275(0-1150)$ & $0(0-288)$ & 0.09 \\
\hline Median length of hospital stay, days (IQR) & $10.0(4.3-21.8)$ & $8.0(4.3-15.8)$ & 0.63 \\
\hline In-hospital complications, yes & $11(45.8)$ & $14(43.8)$ & 0.88 \\
\hline Frail & $2(8.3)$ & $3(6.3)$ & 1.00 \\
\hline ADL-dependent & $1(4.2)$ & $5(16.1)$ & 0.22 \\
\hline iADL-dependent & $8(33.3)$ & 7 (21.9) & 0.38 \\
\hline Risk of malnutrition & $7(29.2)$ & 7 (22.6) & 0.58 \\
\hline Anxiety & $3(12.5)$ & $3(9.7)$ & 1.00 \\
\hline Depression & $10(41.7)$ & $8(25.8)$ & 0.21 \\
\hline
\end{tabular}

Data are expressed as $n(\%)$ unless otherwise specified

Two patients died during hospital admission and were excluded from this table

$S D$ standard deviation, ASA American Society of Anesthesiologists Physical Status Classification System, ${ }^{31} I Q R$ interquartile range, $A D L$ activities of daily living, $i A D L$ instrumental activities of daily living

* Statistically significant difference, $p<0.05$

were caused by low physical activity and deviations in vital signs, mainly blood pressure (Table 3 ).

\section{Physical Activity}

During the first 2 weeks post-discharge, the median daily step count increased from 1600 steps (IQR 500-2930) on day 1 to 3651 steps (IQR 1027-7579) on day 14, without any differences between groups of patients with or without post-discharge complications. The median step count for patients with readmissions was significantly lower than for patients without readmissions on the day before discharge and on day 9 after discharge (Fig. 2). In addition, a threshold violation (step count $<1000$ ) was more often measured in patients who were readmitted compared with patients who were not readmitted (7/12
[58.3\%] vs. 20/39 [51.3\%]; $p=0.02$ ). The rates of patients with threshold violations were similar between the groups with and without post-discharge complications $(52.9 \%$ vs. $55.2 \% ; p=0.25)$.

\section{Vital Signs}

A subset of patients was discharged with a thermometer $(n=38)$, blood pressure/heart rate monitor $(n=37)$, and instructions to manually enter weight into the Connecare application $(n=35)$. A total of $151 / 1231$ vital sign measurements violated the threshold in 25 patients (Table 3 ). These violations were observed in 13/18 (78\%) patients with post-discharge complications and 12/21 (57\%) patients without post-discharge complications $(p=0.30)$ [Fig. 3a]. The rates of patients with threshold violations 
TABLE 2. Details of post-discharge adverse events

\begin{tabular}{|c|c|}
\hline \multicolumn{2}{|l|}{ Categories and classifications } \\
\hline Total post-discharge complications & $24(100)$ \\
\hline Comprehensive Complication Index, median (IQR) & $23.3(8.7-43.5)$ \\
\hline \multicolumn{2}{|l|}{ Highest Clavien-Dindo classification complication } \\
\hline Grade 0 & $3(12.5)$ \\
\hline Grade 1 & $8(33.3)$ \\
\hline Grade 2 & $4(16.7)$ \\
\hline Grade 3A & $3(12.5)$ \\
\hline Grade 3B & $5(20.8)$ \\
\hline Grade 4A & $1(4.2)$ \\
\hline \multicolumn{2}{|l|}{ Type of most serious complication } \\
\hline Infectious & $13(54.2)$ \\
\hline 'Failure to thrive' & $3(12.5)$ \\
\hline Anastomotic leakage & $2(8.3)$ \\
\hline Seroma & $2(8.3)$ \\
\hline Thromboembolic event & $1(4.2)$ \\
\hline Cardiovascular & $1(4.2)$ \\
\hline Drug-induced hypotension & $1(4.2)$ \\
\hline False-positive temperature measurement & $1(4.2)$ \\
\hline \multicolumn{2}{|l|}{ Timing first complication at home } \\
\hline$<14$ days after discharge & $17(70.8)$ \\
\hline 14-30 days after discharge & $3(12.5)$ \\
\hline$>30$ days after discharge & $4(16.7)$ \\
\hline Total of patients readmitted & 13 \\
\hline \multicolumn{2}{|l|}{ Timing hospital readmission } \\
\hline$<14$ days after discharge & $8(61.5)$ \\
\hline 14-30 days after discharge & $1(7.7)$ \\
\hline$>30$ days after discharge & $4(30.8)$ \\
\hline
\end{tabular}

were similar in patients with and without hospital readmissions (Fig. 3b) and per specific vital sign (data not presented).

\section{Patient-Reported Symptoms}

Thirty-three patients were instructed to report their symptoms. Median pain scores and cumulative symptoms did not change over time (Fig. 4). The pain score over the first 2 weeks was significantly higher in patients with postdischarge complications compared with patients without complications (median 3.0 [IQR 1.9-3.8] vs. 0.5 [IQR $0-1.9]$; $p=0.02$ ). However, the rates of patients who experienced a threshold violation for pain were similar between groups with and without post-discharge complications (6 [40\%] vs. $10[56 \%] ; p=0.63)$ and with and without hospital readmissions (3 [38\%] vs. 13 [52\%]; $p=0.13)$.

The symptoms most frequently reported were needing help with activities of daily living (106 times), being less mobile than usual (78 times), and having trouble sleeping/ resting (48 times). In total, 43 threshold violations were caused by nine patients experiencing symptoms of dyspnea, vomiting, vertigo, or fever (Table 3). The percentage of patients who had one or more threshold violations did not differ between patients with and without post-discharge complications (3 [20\%] vs. $6[33 \%] ; p=0.44$ ) or between patients with and without hospital readmissions $(2[25 \%]$ vs. $7[28 \%] ; p=0.25)$.

Data are expressed as $n(\%)$ unless otherwise specified

$I Q R$ interquartile range

TABLE 3. No. of measurements, threshold violations, and patients experiencing threshold violations

\begin{tabular}{llll}
\hline Parameter & No. of measurements & Total no. of threshold violations $(\%)$ & No. of patients with threshold violations \\
\hline Overall & 5379 & $392(7.3)$ & 40 \\
Physical activity & 565 & $168(29.7)$ & 27 \\
Vitals & 1231 & $151(12.3)$ & 25 \\
Temperature & 332 & $29(8.7)$ & 16 \\
Blood pressure & 336 & $62(18.5)$ & 15 \\
Heart rate & 321 & $18(5.6)$ & 7 \\
Weight & 248 & $42(16.9)$ & 6 \\
Patient-reported symptoms & 3583 & $73(2.0)$ & 17 \\
Pain & 271 & $30(11.1)$ & 16 \\
Dyspnea & 276 & $6(2.2)$ & 3 \\
Vomiting & 276 & $4(1.4)$ & 4 \\
Vertigo & 276 & $32(11.6)$ & 5 \\
Fever & 276 & $1(0.4)$ & 1 \\
\hline
\end{tabular}


FIG. 2. Boxplot of daily step count of patients with readmissions (blue) and without hospital readmissions (light green) over time. Statistically significant differences were measured on the day before discharge $(-1, p=0.01)$ and on day $9(p=0.01)$
Daily step count from the day before hospital discharge until 14 days post-discharge for patients with readmission versus patients without readmission

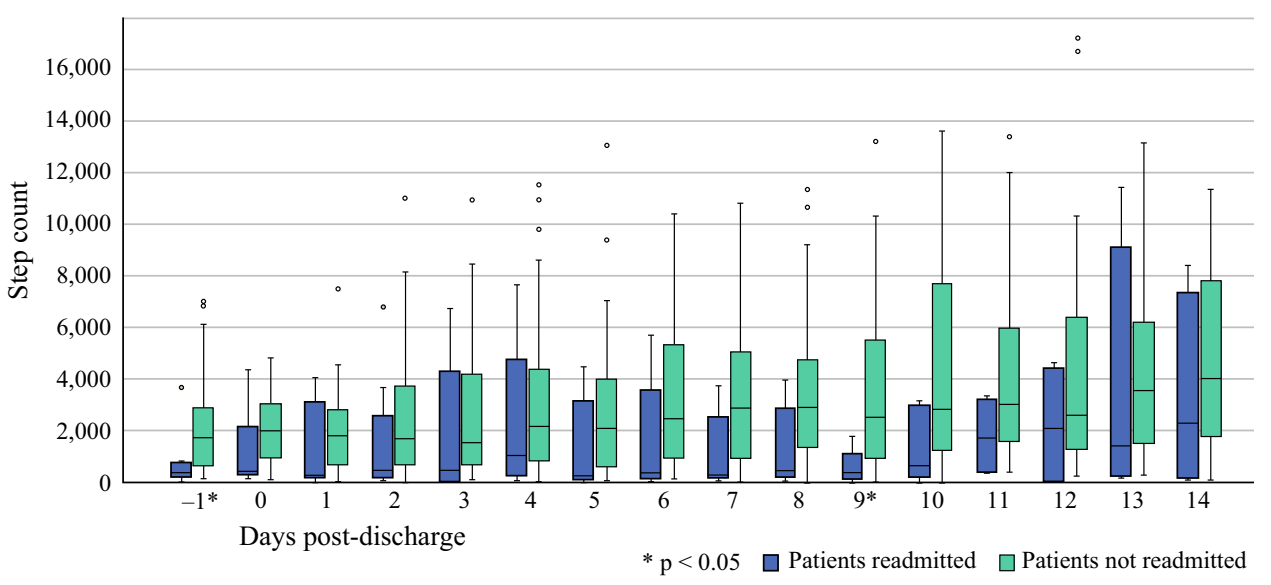

A Vital sign measurement from patients with and without post-discharge complications

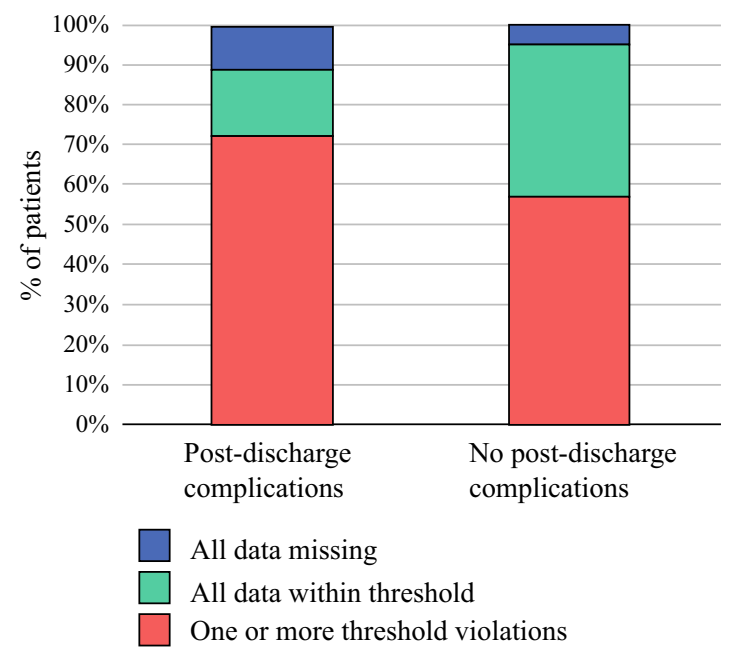

B Vital sign measurement from patients with and without hospital readmissions

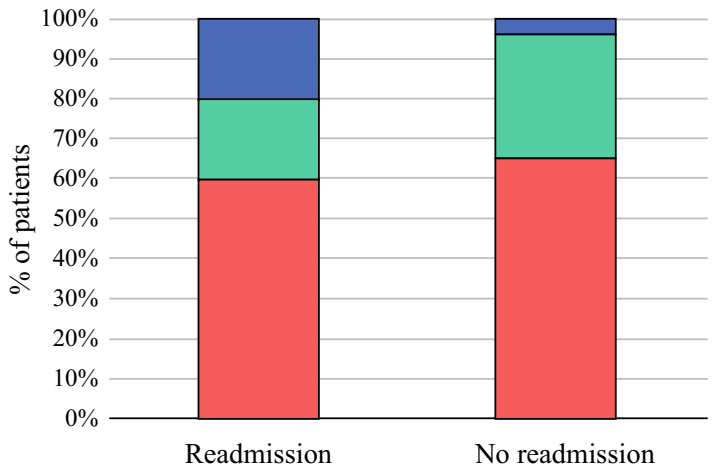

All data missing

All data within threshold

One or more threshold violations

FIG. 3. Percentage of patients with threshold violations, values within the set threshold, and data missing for patients with versus without postdischarge complications (a) and patients with versus without hospital readmission (b). No statistically significant differences were observed

\section{DISCUSSION}

In this observational cohort study, we monitored physical activity, vital signs, and patient-reported symptoms of older patients post-discharge after oncological surgery. No evident relation was found between monitored parameters and adverse events, including complications and readmission. To the best of our knowledge, our study is the first to combine remote home monitoring of physical activity, vital signs, and patient-reported symptoms in older patients after cancer surgery; other studies have only combined monitoring of physical activity with symptoms ${ }^{14}$ or vital signs. ${ }^{12,32}$
The overall postoperative complication rate in our cohort $(69 \%)$ is slightly higher than that reported in other studies after oncogeriatric surgery $(45-60 \%),{ }^{33-35}$ but most studies did not report post-discharge complications. ${ }^{8}$ In our cohort, $24(43 \%)$ patients experienced one or more postdischarge complications and 13 (23\%) were readmitted, which emphasizes the extent of post-discharge events in this population. Most readmissions were due to infections, but some were due to 'failure to thrive', in accordance with data shown in other studies. ${ }^{8,36}$ Complications and readmissions occurred most frequently within 2 weeks after hospital discharge, ${ }^{8}$ which would justify the intensive monitoring of various parameters during this period. We expected to detect these complications by measuring a wide range of monitored parameters, but this primarily 
FIG. 4. Median pain score and cumulative total of symptoms and alarming symptoms (dyspnea, vomiting, vertigo, or fever) per day after hospital discharge

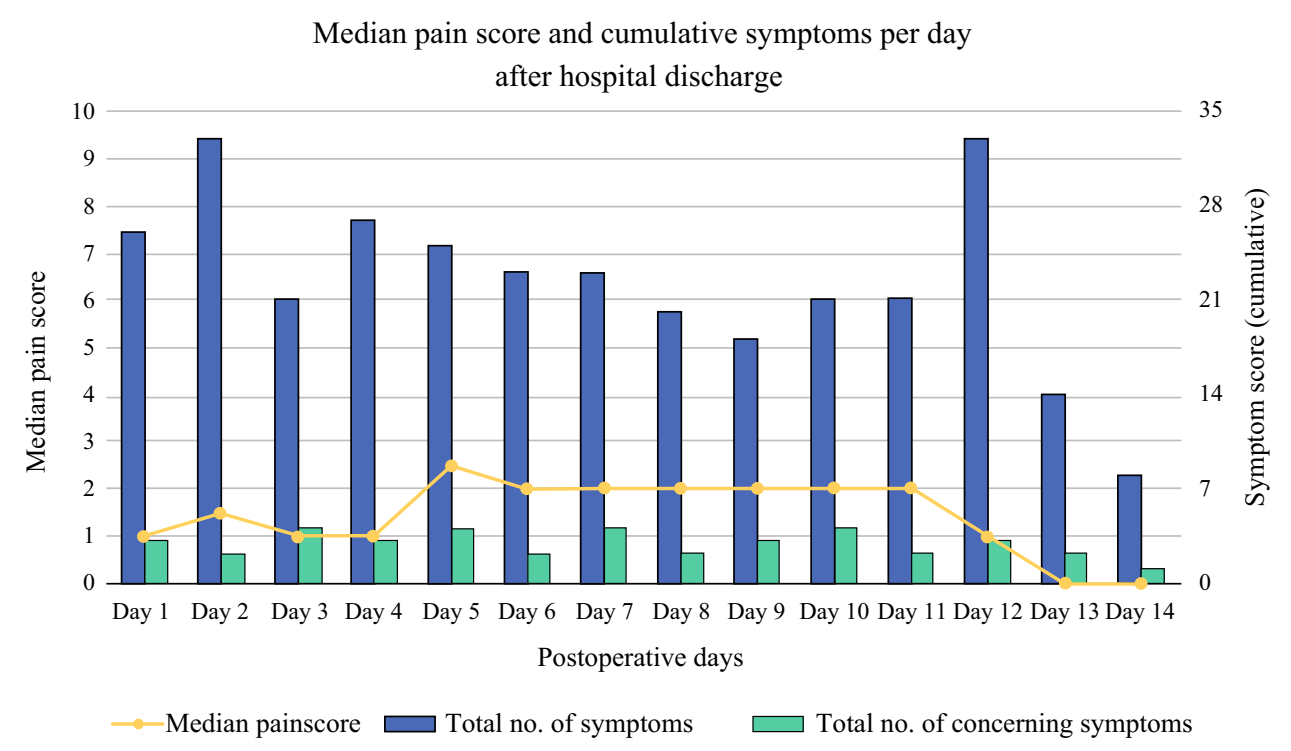

Median pain score and cumulative symptoms per day after hospital discharge

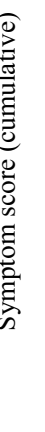
. resulted in a high number of threshold violations, as described previously, ${ }^{37}$ without a clear difference between patients with and without post-discharge events.

Patients who were readmitted had a lower median step count at discharge and in the post-discharge course, and more threshold violations of physical activity compared with patients who were not readmitted. Furthermore, the median pain score of patients with post-discharge complications was significantly higher than that of patients without post-discharge complications. However, the majority of all monitored patients experienced threshold violations of physical activity, vital signs, and patient-reported symptoms in the first 2 weeks after hospital discharge, with similar rates in the groups of patients with and without post-discharge complications and readmissions.

Lower physical activity at discharge was associated with readmissions, in accordance with a previous study where a low inpatient step count resulted in a high risk for 30- and 60-day readmission after metastatic cancer surgery. ${ }^{38}$ Low post-discharge physical activity has already been associated with a complicated postoperative recovery. ${ }^{14,39} \mathrm{We}$ demonstrated that post-discharge physical activity was lower and more often triggered a threshold violation in patients who were readmitted compared with patients who were not. This supports the idea that postoperative physical activity monitoring could function as an indicator of postdischarge complications; ${ }^{39}$ however, it is unclear whether the complications affect physical activity or the physical activity level elevates the risk of having complications.

Regarding vital sign measurements, we hypothesized to encounter more threshold violations in patients with complications, but no differences between patients with and without post-discharge events were found. In addition, not every patient with a complication or readmission had deviations in vital signs. The absence of threshold violations in case of occurrence of complications could be explained by the fact that most complications were Clavien-Dindo grades 0 and 1 (less severe), and that complications in older patients might not be preceded by deviations in vital signs either. ${ }^{40}$ Furthermore, although our study was solely observational, an interventional monitoring study by Metcalf et al. also found that most vital sign threshold violations did not require an intervention. ${ }^{12}$ Finally, thresholds per parameter were based on standardized values from the early warning scores ${ }^{41,42}$ and were not personalized, with the exception of weight loss or gain as a percentage of weight at discharge. If data on patients' preoperative vital signs are gathered, personalized thresholds could provide a higher sensitivity and specificity to detect complications and readmissions.

The severity and presence of patient-reported symptoms in our cohort did not decline over time, as might be expected based on other studies that monitored symptoms post-discharge after cancer surgery. ${ }^{14,15,43}$ Although a difference in median pain score between patients with and without post-discharge complications was demonstrated, the use of threshold violations of patient-reported symptoms did not help us to identify patients with postdischarge complications. This could be explained by the fact that our study did not include any feedback or intervention in response to patient-reported symptoms, unlike other studies in which feedback reduced the symptom burden over time. ${ }^{14,15}$ The action that was most frequently taken when patient-reported symptoms were present in other studies was reinforcement of prescribed treatment or medication, such as pain medication. ${ }^{14-16}$ 
There are several limitations to our study. Although we aimed to understand the post-discharge recovery in all older patients after cancer surgery, our conclusions were constrained by the data we were able to collect. First, more than half of the identified patients undergoing cancer surgery in our hospital did not participate in the study because of perceived mental or technological barriers. ${ }^{21}$ Second, usability problems, technical issues, study dropout, and variable compliance with performance of measurements resulted in missing data. ${ }^{19}$ The main reason for dropout was a complicated postoperative course in 10 of 13 patients, but the dropout rates in patients with or without post-discharge complications did not differ significantly (29\% vs. $19 \% ; p=0.36$; not presented in the Results section). The complexity of surgery could also affect the dropout rate, although it was difficult to compare this with each other due to the high variability and the small samples per surgery type. It should be noted that all patients who were approached for participation in this study were planned for complex surgical procedures in a tertiary referral center for oncological surgery. Post-discharge telemonitoring data were only available for 7 of the 13 patients who dropped out, but the median step count was significantly lower on several early post-discharge days than in the general cohort. This could be explained due to a lower compliance to wearing the Fitbit, or by the fact that patients motivated to improve their activity were more motivated to complete the study. Third, not all parameters were measured in all patients at the start of the study as the system was still under development when the additional vital sign and patient-reported symptom monitoring started. ${ }^{19}$ Finally, other important parameters such as respiration rate and oxygen saturation, validated health questionnaires for patient-reported symptoms, and photographs of surgical sites to enable post-discharge wound monitoring ${ }^{44}$ might have contributed more insight into patients' recovery at home and supported the detection of deviations in recovery.

To address these limitations, future telemonitoring studies should focus on improving accessibility, study inclusion and retention rates, usability, and compliance in older patients after cancer surgery. Our study demonstrates that detecting post-discharge complications following oncogeriatric surgery is complicated and requires more than measurement of a single parameter. Vital sign measurements were not very sensitive or specific for identifying deviations in the post-discharge course of older patients after cancer surgery. It remains to be investigated how this combination affects complication and readmission rates compared with care as usual.

In daily practice, telemonitoring should therefore not be considered a separate tool but rather a supplement to existing perioperative care. In preoperative settings, telemonitoring data could support decision making; for example, whether to start prehabilitation or to proceed with planned surgery. In postoperative care, telemonitoring data should support the existing care for screening, triaging, and scheduling postoperative follow-up. Observed postoperative symptoms could generate automated feedback to patients, which may consist of general nursing advice or early routine or emergency medical consultation and treatment in and outside the hospital. Moreover, post-discharge monitoring after surgery could contribute to better patient-provider communication and promote patient engagement and self-efficacy. ${ }^{45-47}$ Perioperative telemonitoring has the potential to improve 'care as usual' to personalized and efficient care of the future. Reimbursement of telemonitoring is currently hindered by the 'feefor-service' payment model that stimulates production. In order to incentivize telemonitoring as being part of 'care as usual', innovative funding schemes such as bundled payment schemes could be considered. ${ }^{48}$ However, more research on the exact effect on readmission rates and costs in this population is still required.

\section{CONCLUSION}

Detecting and predicting post-discharge complications is complex and multifactorial. Our results confirm this and provide more insight into which parameters could be used to target post-discharge adverse events after oncogeriatric surgery. Low physical activity and higher pain score were associated with post-discharge events and should be used as parameters in future interventional telemonitoring studies.

ACKNOWLEDGMENTS The authors wish to express their gratitude to all colleagues in the Connecare consortium for providing input during the development and support of the IT systems and connected devices used in this study.

AUTHOR CONTRIBUTIONS Study concept and design: LTJ, MMHL, MHMO, GHdB, and BLvL. Acquisition of data: LTJ, MMHL, and MHMO. Analysis and interpretation of data: LTJ, MMHL, GHdB, and BLvL. Drafting of the manuscript: LTJ. Critical revision of the manuscript for important intellectual content: MMHL, MHMO, GHdB, and BLvL.

FUNDING This work was supported by the European Union's Horizon 2020 Research and Innovation Program (Project Grant Number 689802 CONNECARE).

DISCLOSURE None declared.

OPEN ACCESS This article is licensed under a Creative Commons Attribution 4.0 International License, which permits use, sharing, adaptation, distribution and reproduction in any medium or format, as long as you give appropriate credit to the original author(s) and the 
source, provide a link to the Creative Commons licence, and indicate if changes were made. The images or other third party material in this article are included in the article's Creative Commons licence, unless indicated otherwise in a credit line to the material. If material is not included in the article's Creative Commons licence and your intended use is not permitted by statutory regulation or exceeds the permitted use, you will need to obtain permission directly from the copyright holder. To view a copy of this licence, visit http://creativecommons. org/licenses/by/4.0/.

\section{REFERENCES}

1. World Health Organization. Global health aging. Updated 2017. Available at: https://www.nia.nih.gov/sites/default/files/2017-06/ global_health_aging.pdf. Accessed 15 Jun 2020.

2. Ferlay J, Ervik M, Lam F, et al. Global cancer observatory: Cancer today. Lyon: International Agency for Research on Cancer. Updated 2018. Available at: https://gco.iarc.fr/today. Accessed 22 May 2019.

3. Johnston ME, Sussman JJ, Patel SH. Surgical oncology and geriatric patients. Clin Geriatr Med. 2019;35(1):53-63.

4. Ethun CG, Bilen MA, Jani AB, et al. Frailty and cancer: Implications for oncology surgery, medical oncology, and radiation oncology. CA Cancer J Clin. 2017;67(5):362-77.

5. Weerink LBM, Gant CM, van Leeuwen BL, et al. Long-term survival in octogenarians after surgical treatment for colorectal cancer: Prevention of postoperative complications is key. Ann Surg Oncol. 2018;25(13):3874-82.

6. Jia Y, Jin G, Guo S, et al. Fast-track surgery decreases the incidence of postoperative delirium and other complications in elderly patients with colorectal carcinoma. Langenbecks Arch Surg. 2014;399(1):77-84.

7. Regenbogen SE, Cain-Nielsen AH, Norton EC, et al. Costs and consequences of early hospital discharge after major inpatient surgery in older adults. JAMA Surg. 2017;152(5):e170123.

8. Ommundsen N, Nesbakken A, Wyller TB, et al. Post-discharge complications in frail older patients after surgery for colorectal cancer. Eur J Surg Oncol. 2018;44(10):1542-7.

9. Morris MS, Deierhoi RJ, Richman JS, Altom LK, Hawn MT. The relationship between timing of surgical complications and hospital readmission. JAMA Surg. 2014;149(4):348-54.

10. Branowicki PM, Vessey JA, Graham DA, et al. Meta-analysis of clinical trials that evaluate the effectiveness of hospital-initiated postdischarge interventions on hospital readmission. $J$ Healthc Qual. 2017;39(6):354-66.

11. Avery KNL, Richards HS, Portal A, et al. Developing a real-time electronic symptom monitoring system for patients after discharge following cancer-related surgery. BMC Cancer. 2019;19(1):463.

12. Metcalf M, Glazyrine V, Glavin K, et al. The feasibility of a health care application in the treatment of patients undergoing radical cystectomy. J Urol. 2019;201(5):902-8.

13. Graetz I, Anderson JN, McKillop CN, et al. Use of a web-based app to improve postoperative outcomes for patients receiving gynecological oncology care: a randomized controlled feasibility trial. Gynecol Oncol. 2018;150(2):311-7.

14. Sun V, Dumitra S, Ruel N, et al. Wireless monitoring program of patient-centered outcomes and recovery before and after major abdominal cancer surgery. JAMA Surg. 2017;152(9):852-9.

15. Cleeland CS, Wang XS, Shi Q, et al. Automated symptom alerts reduce postoperative symptom severity after cancer surgery: a randomized controlled clinical trial. $J$ Clin Oncol. 2011;29(8):994-1000.

16. Andikyan V, Rezk Y, Einstein MH, et al. A prospective study of the feasibility and acceptability of a web-based, electronic patient-reported outcome system in assessing patient recovery after major gynecologic cancer surgery. Gynecol Oncol. 2012;127(2):273-7.

17. Jonker LT, Haveman ME, de Bock GH, van Leeuwen BL, Lahr $\mathrm{MMH}$. Feasibility of perioperative eHealth interventions for elderly surgical patients: a systematic review. J Am Med Dir Assoc. 2020;21(12):1844-1851.e2.

18. van der Meij E, Anema JR, Otten RH, et al. The effect of perioperative E-health interventions on the postoperative course: a systematic review of randomised and non-randomised controlled trials. PLoS One. 2016;11(7):e0158612.

19. Jonker LT, Plas M, de Bock GH, et al. Remote home monitoring of older surgical cancer patients: perspective on study implementation and feasibility. Ann Surg Oncol. 2021;28(1):67-78.

20. Jonker LT, Hendriks S, Lahr MMH, et al. Postoperative recovery of accelerometer-based physical activity in older cancer patients. Eur J Surg Oncol. 2020;46(11):2083-90.

21. Jonker LT, Lahr MMH, Festen S, et al. Perioperative telemonitoring of older adults with cancer: can we connect them all? $J$ Geriatr Oncol. 2020;11(8):1244-9.

22. Daskivich TJ, Houman J, Lopez M, et al. Association of wearable activity monitors with assessment of daily ambulation and length of stay among patients undergoing major surgery. JAMA Netw Open. 2019;2(2):e187673.

23. Karcioglu O, Topacoglu H, Dikme O, et al. A systematic review of the pain scales in adults: which to use? Am J Emerg Med. 2018;36(4):707-14.

24. Peters LL, Boter H, Buskens E, et al. Measurement properties of the Groningen frailty indicator in home-dwelling and institutionalized elderly people. $J$ Am Med Dir Assoc. 2012;13(6):546-51.

25. Katz S, Ford AB, Moskowitz RW, et al. Studies of illness in the aged. the index of adl: a standardized measure of biological and psychosocial function. JAMA. 1963;185:914-9.

26. Lawton MP, Brody EM. Assessment of older people: self-maintaining and instrumental activities of daily living. Gerontologist. 1969;9(3):179-86.

27. Rubenstein LZ, Harker JO, Salva A, et al. Screening for undernutrition in geriatric practice: developing the short-form mininutritional assessment (MNA-SF). J Gerontol A Biol Sci Med Sci. 2001;56(6):M366-72.

28. Spinhoven P, Ormel J, Sloekers PP, et al. A validation study of the hospital anxiety and depression scale (HADS) in different groups of Dutch subjects. Psychol Med. 1997;27(2):363-70.

29. Clavien PA, Barkun J, de Oliveira ML, et al. The Clavien-Dindo classification of surgical complications: five-year experience. Ann Surg. 2009;250(2):187-96.

30. Clavien PA, Vetter D, Staiger RD, et al. The comprehensive complication index $\left(\mathrm{CCI}{ }^{\circledR}\right)$ : added value and clinical perspectives 3 years "down the line." Ann Surg. 2017;265(6):1045-50.

31. Owens WD, Felts JA, Spitznagel EL, J. ASA physical status classifications: a study of consistency of ratings. Anesthesiology. 1978;49(4):239-243.

32. Wynter-Blyth V, MacKinnon T. Streamlining perioperative care for oesophago-gastric cancer surgery patients using home remote monitoring. Prim Health Care. 2017;27(5):27-31.

33. Badgwell B, Stanley J, Chang GJ, et al. Comprehensive geriatric assessment of risk factors associated with adverse outcomes and resource utilization in cancer patients undergoing abdominal surgery. J Surg Oncol. 2013;108(3):182-6.

34. Kristjansson SR, Nesbakken A, Jordhøy MS, et al. Comprehensive geriatric assessment can predict complications in elderly patients after elective surgery for colorectal cancer: a prospective observational cohort study. Crit Rev Oncol Hematol. 2010;76(3):208-17. 
35. Carli F, Bousquet-Dion G, Awasthi R, et al. Effect of multimodal prehabilitation vs postoperative rehabilitation on 30-day postoperative complications for frail patients undergoing resection of colorectal cancer: a randomized clinical trial. JAMA Surg. 2020;155(3):233-42.

36. Hughes LD, Witham MD. Causes and correlates of 30 day and 180 day readmission following discharge from a medicine for the elderly rehabilitation unit. BMC geriatrics. 2018;18(1):197.

37. Winters BD, Cvach MM, Bonafide CP, et al. Technological distractions (part 2): a summary of approaches to manage clinical alarms with intent to reduce alarm fatigue. Crit Care Med. 2018;46(1):130-7.

38. Low CA, Bovbjerg DH, Ahrendt S, et al. Fitbit step counts during inpatient recovery from cancer surgery as a predictor of readmission. Ann Behav Med. 2018;52(1):88-92.

39. Panda N, Solsky I, Huang EJ, et al. Using smartphones to capture novel recovery metrics after cancer surgery. JAMA Surg. 2019;155(2):1-7.

40. Kim SS, Zenilman ME. The elderly surgical patient. In: ACS surgery: principles and practice. 6th ed. New York, USA: WebMD Inc.; 2008.

41. Subbe CP, Kruger M, Rutherford P, et al. Validation of a modified early warning score in medical admissions. QJM. 2001;94(10):521-6.

42. Dundar ZD, Ergin M, Karamercan MA, et al. Modified early warning score and VitalPac early warning score in geriatric patients admitted to emergency department. Eur J Emerg Med. 2016;23(6):406-12.

43. Gustavell T, Sundberg K, Frank C, et al. Symptoms and self-care following pancreaticoduodenectomy: perspectives from patients and healthcare professionals - foundation for an interactive ICT application. Eur J Oncol Nurs. 2017;26:36-41.

44. Gunter RL, Fernandes-Taylor S, Rahman S, et al. Feasibility of an image-based mobile health protocol for postoperative wound monitoring. J Am Coll Surg. 2018;226(3):277-86.

45. Dawes AJ, Reardon S, Chen VL, et al. Wireless technology to track surgical patients after discharge: a pilot study. Am Surg. 2015;81(10):1061-6.

46. Nijland LMG, van Veen RN, Ruys AT, et al. Feasibility of postoperative home monitoring using video consultation and vital sign monitoring of bariatric patients. Obes Surg. 2020;30(6):2369-74.

47. Onuma AE, Palmer Kelly E, Chakedis J, et al. Patient preferences on the use of technology in cancer surveillance after curative surgery: a cross-sectional analysis. Surgery. 2019;165(4):782-8.

48. Stokes J, Struckmann V, Kristensen SR, et al. Towards incentivising integration: a typology of payments for integrated care. Health Policy. 2018;122(9):963-9.

Publisher's Note Springer Nature remains neutral with regard to jurisdictional claims in published maps and institutional affiliations. 